\title{
Expression of deleted in liver cancer 2 in colorectal cancer and its correlation with clinicopathological parameters
}

\author{
KAI GAO, XIAORONG LI, GUI HU, KAIYAN YANG, BUNING TIAN and YI ZHANG \\ Department of General Surgery, The Third Affiliated Hospital of Central South University, \\ Central South University, Changsha, Hunan 410013, P.R. China
}

Received April 26, 2012; Accepted July 27, 2012

DOI: $10.3892 / \mathrm{ol} .2012 .854$

\begin{abstract}
Deleted in liver cancer 2 (DLC2) has been identified as a tumor suppressor gene. DLC2 is closely related to deleted in liver cancer 1 (DLC1) and is located at chromosome 13q12.3. The expression of DLC2 mRNA has been found in a wide range of cancers, including colorectal cancer (CRC). However, there are no available data on the expression status of DLC2 in Chinese patients with CRC and its correlation with clinicopathological parameters. The aim of this study was to investigate the expression levels of $D L C 2 \mathrm{mRNA}$ and protein in Chinese patients with CRC and the correlation between DLC2 expression and clinicopathological parameters. To this end, real-time PCR, western blotting and immunohistochemistry were employed to detect DLC2 mRNA and protein expression in CRC and pericarcinomatous intestinal tissue (PCIT) specimens, which were obtained from 102 Chinese CRC patients who underwent surgical resection between October 2010 and February 2012. We also analyzed the correlations between DLC2 expression and the clinicopathological parameters of CRC patients. Our results showed that CRC tissues had significantly lower levels of DLC2 mRNA compared with PCITs $(\mathrm{P}<0.05)$; however, the protein expression levels were not significantly different between CRCs and PCITs. The expression levels of $D L C 2$ mRNA and protein were significantly correlated with lymph node metastasis and tumor TNM stage. Additionally, DLC2 mRNA expression levels were also correlated with tumor histopathological degree $(\mathrm{P}<0.05)$. Collectively, our results suggest that the downregulated expression of DLC2 participates in CRC carcinogenesis, invasion and lymph node metastasis. Furthermore, our results imply that DLC2 is be a potential prognostic marker for CRC patients.
\end{abstract}

Correspondence to: Dr Xiaorong Li, Department of General Surgery, The Third Affiliated Hospital of Central South University, Central South University, Tongzipo Road, Changsha, Hunan 410013, P.R. China

E-mail: lixiaorong_x@hotmail.com

Key words: colorectal cancer, deleted in liver cancer 2

\section{Introduction}

Colorectal cancer (CRC) is one of the most common malignant tumors, with 1,200,000 new cases and 608,700 mortalities worldwide annually (1); furthermore, it is the fourth leading cause of cancer-related mortality in China. In recent decades, the incidence of CRC has increased. Despite surgical resection coupled with systemic chemotherapy, approximately half of newly diagnosed CRC patients are likely to succumb to the disease due to tumor recurrence and metastasis (2). The molecular mechanism leading to the development, local invasion and distal metastasis of CRC remains unclear. Thus, the delineation of the mechanisms for colorectal carcinogenesis is of importance, as it may provide novel strategies for diagnosis and prognosis.

Deleted in liver cancer 2 (DLC2), also called STARD 13, has been identified as a tumor suppressor gene and is closely related to deleted in liver cancer 1 (DLC1). DLC2 is located at chromosome 13q12.3 (3). DLC1 and DLC2 proteins share 51\% identity and $64 \%$ similarity at the level of their amino acid sequences. DLC2 protein contains multiple domains, including a sterile $\alpha$ motif (SAM), a RhoGAP and a lipid-binding StARrelated lipid-transfer (START) domain. Previous studies have shown that DLC2 suppressed cytoskeleton reorganization, cell growth, cell migration and transformation in hepatocellular carcinoma (HCC) (4); the START domain of DLC2 has been reported to be involved in mitochondrial localization in hepatoma cells (5) and our previous study indicated that patients with DLC2 underexpression showed a significantly poorer prognosis than those with high DLC2 expression in HCC (6). To date, all studies examining DLC2 expression and its suppressed function have been conducted on HCC. However, $D L C 2 \mathrm{mRNA}$ has been found to be downregulated in a wide range of cancers, including CRC (7), but there have been no available data on the expression levels of DLC2 mRNA and protein in clinical CRC, especially in Chinese patients.

Therefore, in this study we examined the DLC2 mRNA and protein expression in Chinese patients with CRC and evaluated the correlations between DLC2 expression levels and clinicopathological parameters of CRC.

\section{Materials and methods}

Tissue specimens. The study protocol was approved by the Ethics Committee of the Central South University (CSU). 
Fresh samples of CRC tissues and pericarcinomatous intestine tissues (PCITs, $2.0 \mathrm{~cm}$ away from the carcinoma) were obtained from 102 (66 males and 36 females) Chinese patients with primary cancer who underwent surgery at The Third Affiliated Hospital of Central South University between Octorber 2010 and February 2012. Each specimen was divided into two parts; one was immediately frozen in liquid nitrogen and stored at $-80^{\circ} \mathrm{C}$ for real-time PCR and western blot analysis, the other was fixed in $4 \%$ paraformaldehyde for immunohistochemistry. The median age of the patients was 55.43 years, ranging from 26 to 78 years. All specimens obtained from surgical resection were confirmed by pathological examination. Written informed consent was obtained from each patient prior to the study.

Real-time PCR. Total RNA was extracted from specimens using TRIzol reagent (Invitrogen, Carlsbad, CA, USA) according to the manufacturer's instructions. Aliquots of $5 \mu \mathrm{g}$ RNA from each sample were reverse transcribed. Quantitative analysis of DLC2 mRNA expression using the SYBR Green method was performed using the ABI 7300 real-time PCR system. The sequences of the primers were as follows: DLC2 5'-GTCAATGGGCTCCAAGAGGTAG-3' (forward) and 5'-TGATTAGGAGATGGAAAGGTGGA-3' (reverse); GAPDH (endogenous control): 5'-CTGCACCACCAACTG CTTAG-3' (forward); 5'-AGGTCCACCACTGACACGTT-3' (reverse). A $25-\mu 1$ reaction mixture containing $1 \mu \mathrm{l}$ cDNA template, $12.5 \mu \mathrm{l} \mathrm{SYBR}$ Master mix and $0.20 \mu \mathrm{l}$ of each primer was amplified using the following thermal parameters: denaturing at $95^{\circ} \mathrm{C}$ for $10 \mathrm{~min}$ and 45 cycles of the amplification step (denaturation at $95^{\circ} \mathrm{C}$ for $15 \mathrm{sec}$, annealing and extension at $60^{\circ} \mathrm{C}$ for $1 \mathrm{~min}$ ). All amplification reactions were performed in triplicate and the averages of the threshold cycles (Cts) were used to interpolate curves using 7300 System SDS software. The results were expressed as the ratio of $D L C 2$ mRNA to GAPDH mRNA, and the results of each sample was compared against one control sample to eliminate the differences between the PCR plates.

Western blot analysis. Protein was extracted from the specimens using the fierce RIPA lysis buffer (Beyotime Biotech, Jiangsu, China) following the manufacturer's instructions. Briefly, an equal amount (35 $\mu \mathrm{g})$ of the total protein was separated by SDS-polyacrylamide gel electrophoresis (SDS-PAGE; Beyotime Biotech) and then transferred onto a polyvinylidene difluoride (PVDF; Pall, NY, USA) membrane using standard procedures. The membranes were immunoblotted with antibodies against DLC2 (rabbit polyclonal antibody; Sigma, St. Louis, MO, USA), followed by a horseradish peroxidase-conjugated secondary antibody. The washes were repeated before the membranes were detected using ECL system (KPL Biosciences, Gaithesburg, MD, USA). Band intensities were analyzed densitometrically using the Image $\mathrm{J}$ software.

Immunohistochemistry. All the specimens were immunohistochemically evaluated. In brief, tissue sections of $3-\mu \mathrm{m}$ thickness were cut and baked at $60^{\circ} \mathrm{C}$, deparaffinized in xylene and rehydrated through graded ethanol. Next, 3\% hydrogen peroxide and methanol were applied to block the endogenous peroxidases and sections were subjected to heat-induced antigen

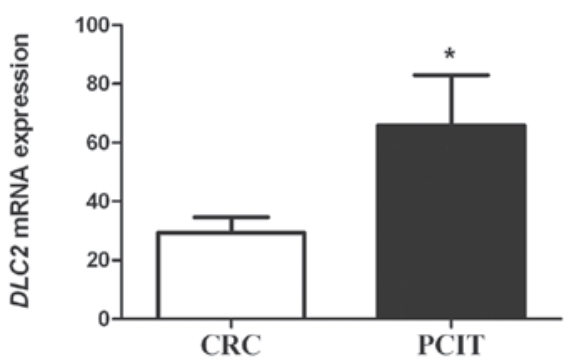

Figure 1. Expression of DLC2 mRNA in CRC and PCIT. The mRNA expression in CRC was significantly lower than that in PCIT (mean $\pm \mathrm{SEM}$, $\mathrm{n}=102),{ }^{*} \mathrm{P}<0.05$ compared with CRC. DLC2, deleted in liver cancer 2; CRC, colorectal cancer; PCIT, pericarcinomatous intestinal tissue.

retrieval in $0.01 \mathrm{M}$ citrate buffer $(\mathrm{pH}=6.0)$. The samples were incubated overnight with primary antibodies against DLC2 at a 1:400 dilution (rabbit polyclonal antibody; Sigma). Then, the samples were treated with MaxVision TM HRP-Polymer anti-Mouse/Rabbit IHC kit (KIT-5020, MaxVision, Shenzen, China) and 3,3'-diaminobenzidine tetrahydrochloride (DAB). Counterstaining was performed with $0.5 \%$ hematoxylin.

Statistical analysis. Statistical analysis was performed using SPSS software (version 17.0; SPSS, Inc., Chicago, IL, USA). Data are expressed as mean \pm SD and, for clarity, as mean \pm SEM in figures. The Student's t-test was used to analyze the correlation betwen the expression of DLC 2 in CRC and PCIT. The non-parametric Mann-Whitney U test was used to analyze the correlations between the expression of DLC2 and clinicopathological variables. $\mathrm{P}<0.05$ was considered to indicate a statistically significant result.

\section{Results}

Expression of DLC2 $\mathrm{mRNA}$ in CRC and PCIT. The expression of DLC2 mRNA was detected in all CRC and PCIT specimens using real-time PCR. The relative expression of DLC 2 mRNA

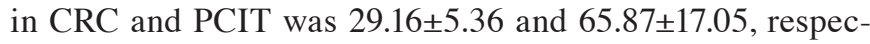
tively. The mRNA expression in CRC tissues was significantly lower than that in PCIT ( $\mathrm{P}=0.037$; Fig. 1).

Expression of DLC2 protein in CRC and PCIT. The protein expression was detected in all CRC and PCIT specimens using western blotting. The relative expression of DLC2 protein in CRC and PCIT was 2.29 \pm 0.61 and $1.66 \pm 0.65$, respectively. There was no significant difference between CRC and PCIT. In addition, to identify the DLC2 protein expression status, immunohistochemical staining was also employed. The result was consistent that of the western blot analysis (Fig. 2).

Correlations between DLC2 expression levels and clinicopathological parameters of CRC. The distribution pattern of DLC2 was determined by dividing the expression levels of DLC2 mRNA and protein in the subgroups by clinicopathological parameters. A significant difference was found between DLC2 expression levels in CRC with or without lymph node metastasis $(\mathrm{P}=0.021$ and 0.028 , respectively); both DLC2 mRNA and protein expression levels in CRC with lymph node metastasis were lower than those in CRC cases 
A

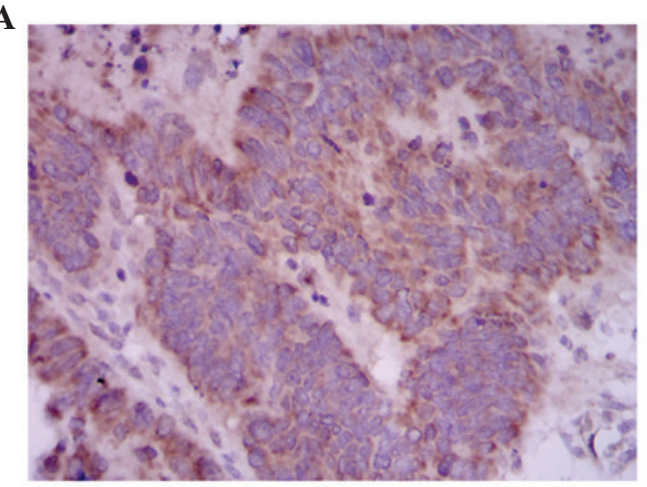

C

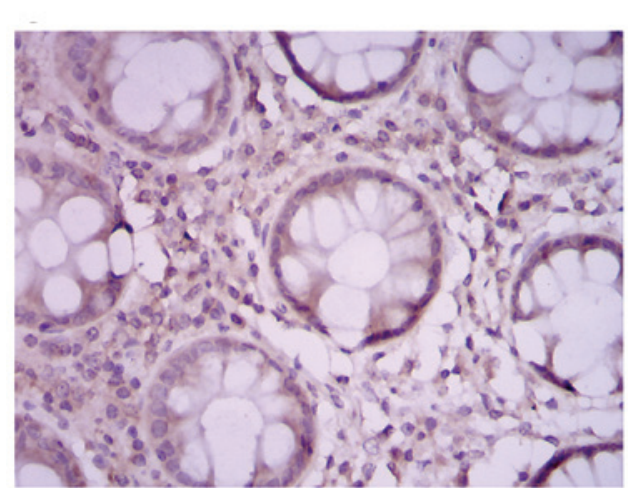

B

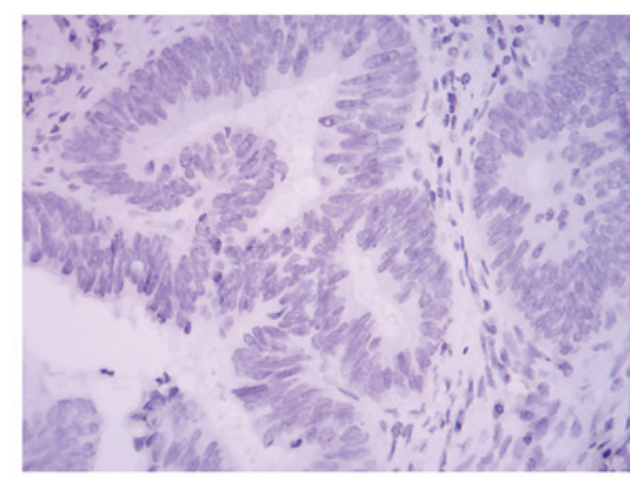

D

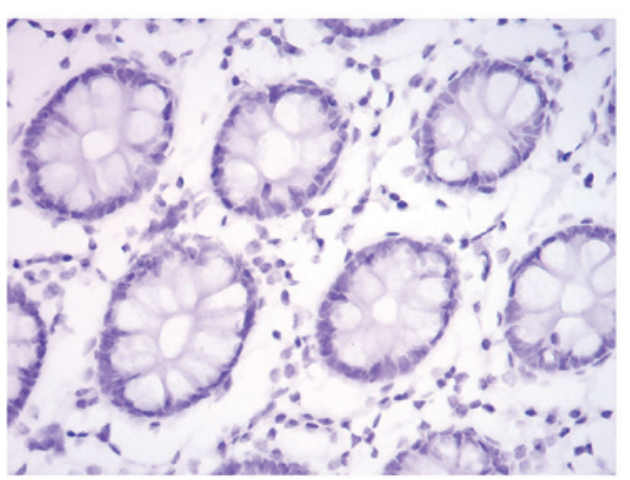

Figure 2. Expression of DLC2 was detected in CRC and PCIT deparaffinized specimens by immunohistochemistry. (A) Positive expression of DLC2 in CRC. (B) Negative expression of DLC2 in CRC. (C) Positive expression of DLC2 in PCIT. (D) Negative expression of DLC2 in PCIT. DLC2, deleted in liver cancer 2; CRC, colorectal cancer; PCIT, pericarcinomatous intestinal tissue.
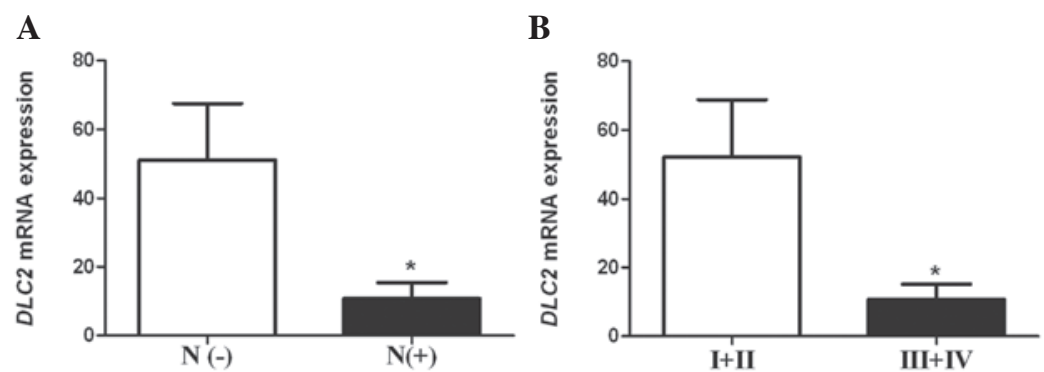

Figure 3. Correlation between $D L C 2$ mRNA expression and lymph node metastasis and between $D L C 2$ mRNA expression and tumor TNM stage. (A) N (-), lymph node metastasis-negative (mean \pm SEM, $\mathrm{n}=50$ ); $\mathrm{N}(+)$, lymph node metastasis-positive (mean $\pm \mathrm{SEM}, \mathrm{n}=52$ ); "P<0.05 compared with $\mathrm{N}(-)$. (B) I+II, TNM stage I and II (mean \pm SEM, $n=49$ ); III+IV, TNM stage III and IV (mean \pm SEM, $n=53$ ); ${ }^{\prime} \mathrm{P}<0.05$ compared with $\mathrm{I}+\mathrm{II}$. DLC2, deleted in liver cancer 2.
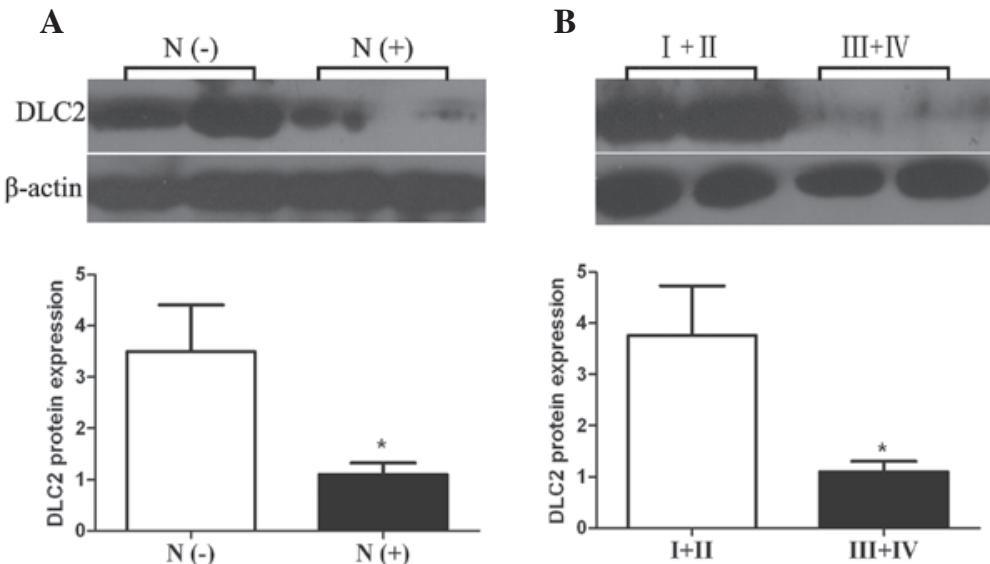

Figure 4. Correlation between DLC2 protein expression and lymph node metastasis and between DLC2 protein expression and tumor TNM stage. (A) N (-), lymph node metastasis-negative (mean $\pm \mathrm{SEM}, \mathrm{n}=50$ ); $\mathrm{N}(+)$, lymph node metastasis-positive (mean $\pm \mathrm{SEM}, \mathrm{n}=52$ ); " $\mathrm{P}<0.05$ compared with $\mathrm{N}(-)$. (B) I+II, TNM stage I and II (mean \pm SEM, $\mathrm{n}=49$ ); III+IV, TNM stage III and IV (mean $\pm \mathrm{SEM}, \mathrm{n}=53$ ); ${ }^{\circ} \mathrm{P}<0.05$ compared with I+II. DLC2, deleted in liver cancer 2. 
Table I. Correlation between expression levels of DLC2 and clinicopathological parameters.

\begin{tabular}{|c|c|c|c|}
\hline \multirow[b]{2}{*}{ Variable } & \multirow{2}{*}{$\begin{array}{l}\text { No. of } \\
\text { patients }\end{array}$} & \multicolumn{2}{|c|}{ P-value } \\
\hline & & $D L C 2$ mRNA & DLC2 protein \\
\hline Gender & & 0.429 & 0.288 \\
\hline Male & 66 & & \\
\hline Female & 36 & & \\
\hline Age (years) & & 0.625 & 0.420 \\
\hline$<60$ & 60 & & \\
\hline$\geq 60$ & 42 & & \\
\hline Tumor site & & 0.528 & 0.918 \\
\hline Colon & 45 & & \\
\hline Rectum & 57 & & \\
\hline Tumor size (cm) & & 0.457 & 0.425 \\
\hline$<5.0$ & 52 & & \\
\hline$\geq 5.0$ & 50 & & \\
\hline Tumor depth & & 0.876 & 0.354 \\
\hline $\begin{array}{l}\text { Limited to } \\
\text { muscular layer }\end{array}$ & 66 & & \\
\hline $\begin{array}{l}\text { Serosa and } \\
\text { adjacent fat }\end{array}$ & 36 & & \\
\hline $\begin{array}{l}\text { Lymph node } \\
\text { metastasis }\end{array}$ & & $0.021^{\mathrm{a}}$ & $0.028^{\mathrm{a}}$ \\
\hline Negative & 50 & & \\
\hline Positive & 52 & & \\
\hline Distant metastasis & & 0.338 & 0.233 \\
\hline Absent & 94 & & \\
\hline Present & 8 & & \\
\hline Tumor TNM stage & & $0.020^{\mathrm{a}}$ & $0.026^{\mathrm{a}}$ \\
\hline I and II & 49 & & \\
\hline III and IV & 53 & & \\
\hline $\begin{array}{l}\text { Tumor } \\
\text { differentiation }\end{array}$ & & $0.004^{\mathrm{a}}$ & 0.080 \\
\hline Well-moderate & 76 & & \\
\hline Poor & 26 & & \\
\hline CEA (ng/ml) & & 0.903 & 0.271 \\
\hline$\geq 5$ & 41 & & \\
\hline$<5$ & 61 & & \\
\hline
\end{tabular}

${ }^{\mathrm{a}} \mathrm{P}<0.05$. DLC2, deleted in liver cancer 2; CEA, carcinoembryonic antigen.

without lymph node metastasis (Figs. 3A and 4A). DLC2 mRNA and protein expression levels of CRC of tumor TNM stage III and IV also showed lower expression levels than in CRC cases of stage I and II ( $\mathrm{P}=0.020$ and 0.026 , respectively; Figs. 3B and 4B). In addition, the expression status of $D L C 2$ mRNA was also correlated with tumor histopathological degree; poorly differentiated CRC cases had a lower expression level of DLC2 than well- and moderately differentiated CRC ( $\mathrm{P}=0.004$; Fig. 5). However, there was no significant association between the expression levels of DLC2 and other

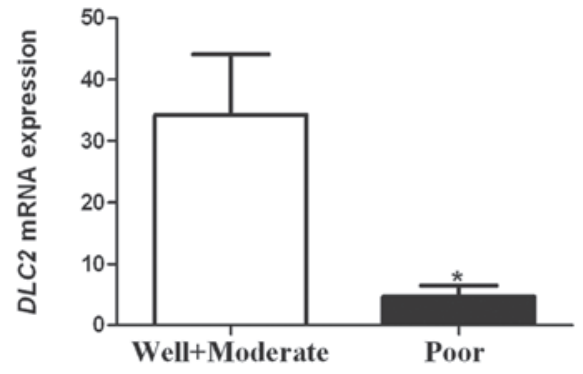

Figure 5. Correlation between $D L C 2$ mRNA expression and tumor histopathological degree. Poor, poorly differentiated CRC (mean \pm SEM, $n=26$ ); Well + moderate, well- and moderately differentiated CRC (mean \pm SEM, $\mathrm{n}=76)$. ${ }^{*} \mathrm{P}<0.05$ compared with well + moderate. DLC2, deleted in liver cancer 2; CRC, colorectal cancer.

clinicopathological parameters, including gender, age, tumor size, tumor site, depth of cancer invasion, distant metastasis and blood levels of carcinoembryonic antigen (CEA; Table I).

\section{Discussion}

Although DLC2 has been identified as a tumor suppressor gene, the expression levels of $D L C 2 \mathrm{mRNA}$ and protein, especially in clinical CRC of Chinese patients, have not been studied. The present study indicates that the expression level of DLC2 mRNA was significantly lower in CRC than in PCIT; however, the protein expression level of DLC2 was not significantly different in CRC compared with PCIT in Chinese patients. This difference between mRNA and protein may be due to post-transcriptional control. With regard to human neoplasia, Ullmannova and Popescu reported that the DLC2 mRNA level was downregulated in lung, ovarian, renal, breast, uterine, gastric, colon and rectal tumors and that the expression ratios of DLC2 in colon and rectal cancer were 46 and $61 \%$, respectively (7). Our real-time PCR results showed that $D L C 2$ mRNA was also underexpressed and the expression ratios of $D L C 2$ mRNA were 70 and $62 \%$ in colon and rectal cancer, respectively. This result is higher than that reported by Ullmannova and Popescu; the reason for this difference may be differences between ethnicities. Also, the low expression of DLC2 has been confirmed to be involved in the invasion and migration of HCC cells (4). DLC2 protein has been reported to inhibit the activity of Raf-1-ERK1/2-p70S6K via its RhoGAP function, resulting in the suppression of cell growth (8). DLC2 has been found to modulate angiogenic responses in vascular endothelial cells by regulating cell attachment and migration (9). Consistent with previous studies, the present study showed that DLC2 mRNA was downregulated in CRC, which indicates that DLC2 participates in colorectal carcinogenesis and plays an important role in the invasion and metastasis of CRC.

To investigate how DLC2 affects colorectal carcinogenesis, we evaluated the correlations between DLC2 expression levels, including mRNA and protein, and clinicopathological parameters of CRC. Our results revealed that the expression levels of $D L C 2$ mRNA and protein were frequently with lymph node metastasis and the DLC2 expression levels in CRC with lymph node metastasis were lower than those in CRC without lymph node metastasis. This result indicates that DLC2 is a tumor suppressor, whose downregulated 
expression stimulates the lymph node metastasis of CRC. This implies that DLC2 has a role in suppressing CRC lymph node metastasis. Our previous study showed that a lower expression level of DLC2 was associated with higher expression level of RhoA in HCC (6). In addition, other studies have shown that DLC2 exhibited its tumor suppressor functions by means of the inhibition of RhoA activity (4); a higher level expression of RhoA has been detected in several types of cancer, including bladder, testicular, ovarian, colon, breast and lung cancer (10-13); Takami et al demonstrated that the activity of RhoA was correlated with lymph node metastasis in human CRC (14). Accordingly, we hypothesized that the downregulation of DLC2 and upregulation of RhoA may be essential in CRC progression, especially in the process of CRC lymph node metastasis. To confirm this hypothesis, investigations into the correlation between DLC2 and RhoA are anticipated.

Notably, in the present study, the mRNA and protein expression levels of DLC2 were also correlated with CRC tumor TNM stage. Our results revealed that the $D L C 2 \mathrm{mRNA}$ and protein expression levels in CRC of TNM stages III and IV were lower than those in CRCs of stage I and II. This result implies that downregulated mRNA and protein expression levels of DLC2 were correlated with advanced stage of CRC. Additionally, the mRNA expression level was correlated with tumor histopathological degree of CRC. It is well known that the TNM staging system of tumors is the gold standard for evaluating prognosis in patients with CRC. It has been reported that the five-year survival rates of CRC patients who underwent surgical resections were 94.1, 80.2, 61.7 and $23.2 \%$ with tumors of TNM stages I to IV, respectively (15); additionally, low tumor histopathological differentiation was also associated with poor survival (16). Thus, it is reasonable to assume that the downregulated expression of DLC2 is correlated with a poor prognosis of CRC patients. To test this assumption, we intend to continue the follow-up of these patients in our further research.

To the best of our knowledge, this is the first study to report DLC2 mRNA and protein expression levels in Chinese CRC patients and the downregulated expression levels of $D L C 2$ mRNA and protein are associated with lymph node metastasis and tumor TNM stage in CRC. The DLC2 mRNA expression level is also correlated with tumor histopathological degree. Although a number of diagnostic advances have been made, it remains difficult to accurately diagnose lymph nodes metastasis and assess the prognosis of CRC. In this study, we found that the downregulated expression levels of $D L C 2$ mRNA and protein were correlated with lymph node metastasis and tumor TNM stage. Furthermore, the underexpression of $D L C 2$ mRNA was also associated with tumor histopathological degree of CRC. Therefore, the present study demonstrates that DLC2 may not only participate in CRC carcinogenesis, invasion and lymph node metastasis, but may also be a potential indicator for the presence of lymph nodes metastasis in CRC and a prognostic marker for CRC patients.

\section{Acknowledgements}

This study was supported by the Planned Science and Technology Project of Hunan Province, China (no. 22010FJ3087) and the Chinese National Science Foundation (no. 81172298). We would like to thank Dr Ren Guo for assistance with experiments and manuscript reviewing and formatting.

\section{References}

1. Jemal A, Bray F, Center MM, Ferlay J, Ward E and Forman D: Global cancer statistics. CA Cancer J Clin 61: 69-90, 2011.

2. Wilke HJ and Van Cutsem E: Current treatments and future perspectives in colorectal and gastric cancer. Ann Oncol 14 (Suppl 2): ii49-ii55, 2003.

3. Ching YP, Wong CM, Chan SF, et al: Deleted in liver cancer (DLC) 2 encodes a RhoGAP protein with growth suppressor function and is underexpressed in hepatocellular carcinoma. J Biol Chem 278: 10824-10830, 2003.

4. Leung TH, Ching YP, Yam JW, et al: Deleted in liver cancer 2 (DLC2) suppresses cell transformation by means of inhibition of RhoA activity. Proc Natl Acad Sci USA 102: 15207-15212, 2005.

5. Ng DC, Chan SF, Kok KH, et al: Mitochondrial targeting of growth suppressor protein DLC2 through the START domain. FEBS Lett 580: 191-198, 2006.

6. Xiaorong L, Wei W, Liyuan Q and Kaiyan Y: Underexpression of deleted in liver cancer 2 (DLC2) is associated with overexpression of RhoA and poor prognosis in hepatocellular carcinoma. BMC Cancer 8: 205, 2008.

7. Ullmannova V and Popescu NC: Expression profile of the tumor suppressor genes DLC-1 and DLC-2 in solid tumors. Int J Oncol 29: 1127-1132, 2006

8. Leung TH, Yam JW, Chan LK, Ching YP and Ng IO: Deleted in liver cancer 2 suppresses cell growth via the regulation of the Raf-1-ERK1/2-p70S6K signalling pathway. Liver Int 30: 1315-1323, 2010.

9. Lin Y, Chen NT, Shih YP, Liao YC, Xue L and Lo SH: DLC2 modulates angiogenic responses in vascular endothelial cells by regulating cell attachment and migration. Oncogene 29: 3010-3016, 2010.

10. Jaffe $A B$ and Hall A: Rho GTPases in transformation and metastasis. Adv Cancer Res 84: 57-80, 2002.

11. Kamai $\mathrm{T}$, Arai $\mathrm{K}$, Tsujii $\mathrm{T}$, Honda $\mathrm{M}$ and Yoshida $\mathrm{K}$ : Overexpression of RhoA mRNA is associated with advanced stage in testicular germ cell tumour. BJU Int 87: 227-231, 2001.

12. Fritz G, Just I and Kaina B: Rho GTPases are over-expressed in human tumors. Int J Cancer 81: 682-687, 1999.

13. Kamai T, Tsujii T, Arai K, et al: Significant association of Rho/ ROCK pathway with invasion and metastasis of bladder cancer. Clin Cancer Res 9: 2632-2641, 2003.

14. Takami Y, Higashi M, Kumagai S, et al: The activity of RhoA is correlated with lymph node metastasis in human colorectal cancer. Dig Dis Sci 53: 467-473, 2008.

15. Luo Y, Cui J, Chen C, et al: Clinical outcomes after surgical resection of colorectal cancer in 1,294 patients. Hepatogastroenterology 59: 1398-1402, 2011.

16. Gill S, Loprinzi CL, Sargent DJ, et al: Pooled analysis of fluorouracil-based adjuvant therapy for stage II and III colon cancer: who benefits and by how much? J Clin Oncol 22: 1797-1806, 2004. 